\title{
The Promise and Challenge of Therapeutic MicroRNA Silencing in Diabetes and Metabolic Diseases
}

\author{
Praveen Sethupathy ${ }^{1,2}$
}

Published online: 25 April 2016

(C) The Author(s) 2016. This article is published with open access at Springerlink.com

\begin{abstract}
MicroRNAs (miRNAs) are small, non-coding, RNA molecules that regulate gene expression. They have a long evolutionary history and are found in plants, viruses, and animals. Although initially discovered in 1993 in Caenorhabditis elegans, they were not appreciated as widespread and abundant gene regulators until the early 2000 s. Studies in the last decade have found that miRNAs confer phenotypic robustness in the face of environmental perturbation, may serve as diagnostic and prognostic indicators of disease, underlie the pathobiology of a wide array of complex disorders, and represent compelling therapeutic targets. Preclinical studies in animal models have demonstrated that pharmacologic manipulation of miRNAs, mostly in the liver, can modulate metabolic phenotypes and even reverse the course of insulin resistance and diabetes. There is cautious optimism in the field about miRNA-based therapies for diabetes, several of which are already in various stages of clinical trials. This review will highlight both the promise and the most pressing challenges of therapeutic miRNA silencing in diabetes and related conditions.
\end{abstract}

Keywords MicroRNA · Therapeutics · Anti-sense oligonucleotide $\cdot$ Diabetes $\cdot$ Metabolic disease $\cdot$ Aptamer

This article is part of the Topical Collection on Pathogenesis of Type 2 Diabetes and Insulin Resistance

Praveen Sethupathy

praveen_sethupathy@med.unc.edu

1 Department of Genetics, School of Medicine, UNC Chapel Hill, Chapel Hill, NC 27599, USA

2 Lineberger Comprehensive Cancer Center, School of Medicine, UNC Chapel Hill, Chapel Hill, NC 27599, USA

\section{Introduction}

Recent advances in sequencing technology have unveiled the diversity and complexity of both small and long non-coding RNAs (ncRNAs). Small RNAs, including short interfering RNAs (siRNAs) and microRNAs (miRNAs), mediate posttranscriptional gene silencing by RNA interference (RNAi), which was designated as the "Breakthrough of the Year" by the journal Science in 2002 and was the discovery for which the Nobel Prize in Physiology or Medicine was awarded in 2006. Furthermore, also in 2006, two specific miRNAs were selected as "Molecule of the Year" by the International Society for Molecular and Cell Biology and Biotechnology Protocols and Researchers (ISMCBBPR) based on the discovery that their tissue expression levels can accurately predict lung cancer prognosis. Since then, miRNAs have emerged as candidate circulating biomarkers of physiologic status [1]; potent regulators of diverse biological pathways from differentiation [2] to energy metabolism [3]; adaptive responders to environmental stimuli such as dietary nutrients [4], microbes [5], and pollutants [6]; inter-organ messengers in health and disease [7•]; and potential therapeutic targets in a wide array of disorders [8]. One context in which the role of miRNAs is increasingly appreciated is type 2 diabetes (T2D) [9], as well as related metabolic conditions such as obesity and hyperlipidemia.

The discovery of the importance of miRNAs to the etiology of these diseases was greatly facilitated by the advent of antisense technology for the study of miRNAs in vivo [10]. AntimiRNA oligonucleotides (AMOs), also referred to as antagomiRs, are chemically modified stretches of nucleic acids that are partially or wholly complementary to specific target miRNAs. AMOs bind their target miRNAs with high specificity and potently suppress their molecular functions. In order to be effective in vivo, AMOs must be stable in circulation and readily taken up by tissues, which are features that are 
conferred by several different types of chemical modifications. The different AMO chemistries have been summarized previously [11]. One popular modification is the locked nucleic acid (LNA), wherein one or more monomers along the length of an AMO are locked in a specific conformation that enhances base stacking and increases the stability of base pairing with its target miRNA by $2-8{ }^{\circ} \mathrm{C}$ per locked monomer. The locked conformation is also refractory to digestion by exo- and endo-nucleases, thereby increasing the stability of AMOs in circulation. AMOs are increasingly used for in vivo research studies of miRNA function and are being developed for potential therapeutic use as well [12]. For example, LNA-mediated inhibition of miR-122 (initially tested in non-human primate models [13]) in patients with chronic hepatitis $\mathrm{C}$ was shown in phase $2 \mathrm{a}$ clinical trials to be non-immunogenic and highly effective at reversing the course of the disease by promoting viral clearance [14]. The success of LNA-anti-miR-122 (miravirsen) has generated sustained interest in the use of AMOs for in vivo silencing of additional miRNAs in other health conditions, most notably cancers and metabolic diseases.

\section{Promising Pre-clinical Examples of Therapeutic MicroRNA Silencing in Metabolic Disease}

Numerous studies in the last few years have used AMOs for therapeutic silencing of at least ten different miRNAs in animal models to study their roles in diabetes, as well as related conditions including obesity, hyperlipidemia, and insulin resistance, and their potential as therapeutic targets (Table 1).

Below are highlighted specific examples in obesity/T2D, as well as hyperlipidemia.

\section{Obesity and Type 2 Diabetes}

Obesity and T2D are complex metabolic disorders that affect hundreds of millions of people worldwide, have no cure, and are associated with numerous debilitating co-morbidities. At the physiologic level, T2D is characterized by resistance to insulin action in peripheral tissues such as the liver, adipose, and muscle and/or failure of pancreatic beta cells to produce/ secrete enough insulin to meet metabolic demands, resulting in chronically elevated blood glucose levels (hyperglycemia) and numerous downstream complications. Both obesity and T2D are growing epidemics and critically require new and effective preventative and therapeutic strategies.

In 2011, Trajkovski et al. [18••] published a seminal study on therapeutic silencing of miRNAs in a mouse model of obesity/T2D. Specifically, they administered 2'-O-methyl modified AMOs by tail-vein injection to silence both miR103 and miR-107 in the livers and adipose of $o b / o b$ mice as well as wild-type C57BL/6J mice on high-fat diet (HFD) for 12 weeks. The effects of the AMO on miR-103/107 levels in other tissues were not reported. They found in both animal models that AMO-mediated suppression of miR-103 and miR-107, which are elevated in the livers of rodents and humans with insulin resistance, significantly alleviate hyperglycemia in large part by promoting insulin signaling in the liver and adipose. Specifically, while glucose uptake by muscle was not affected, glucose production and glycogen content in the liver, as well as adipocyte size, were significantly reduced. Overall, the findings from this study revealed that both miR-103 and miR-107 are key negative regulators of insulin signaling and also suggested that they are novel candidate therapeutic targets for T2D. More recent studies have shown that miR-107 levels may be regulated by inflammatory cues [26] in addition to dietary lipids [27] and that miR-107 is involved in the control of circadian rhythm [27], indicating that both miR-103 and miR-107 likely have broader roles in T2D etiology than through regulation of insulin signaling alone. In April 2015, Regulus Therapeutics announced that a GalNAc-conjugated AMO against miR-103/107 (RG-125) was selected by AstraZeneca for clinical development for the treatment of hepatic steatosis in patients with T2D and/or
Table 1 Pre-clinical animal studies of anti-miRNA oligonucleotide (AMO) based therapeutics in diabetes and related metabolic conditions

\begin{tabular}{lll}
\hline MicroRNA & Metabolic phenotype/disease & References \\
\hline let-7 & Type 2 diabetes & Frost and Olson, PNAS [15] \\
miR-34 & Obesity/type 2 diabetes & Fu et al., PNAS [16] \\
& & Choi et al., Aging Cell [17] \\
miR-103/107 & Obesity/type 2 diabetes & Trajkovski et al., Nature [18••] \\
miR-802 & Obesity/type 2 diabetes & Kornfeld et al., Nature [19] \\
miR-208a & Obesity/type 2 diabetes & Grueter et al., Cell [20] \\
miR-181a & Insulin resistance/type 2 diabetes & Zhou et al., Diabetologia [21] \\
miR-379 & Obesity/hyperlipidemia & deGuia et al., EMBO Journal [22] \\
miR-146b & Obesity & Ahn et al., EMBO Molecular Medicine [23] \\
miR-24 & Steatosis/hyperlipidemia & Ng et al., Hepatology [24] \\
miR-29 & Hyperlipidemia & Kurtz et al., Scientific Reports [25] \\
\hline
\end{tabular}


insulin resistance (http://ir.regulusrx.com/releasedetail.cfm? ReleaseID $=905305$ ).

In 2013, Choi et al. [17] used 2'-O-methoxyethyl phosphorothioate-modified AMOs to silence expression and activity of miR-34a (AMO34) in a mouse model of obesity (8-12-week-old C57BL6/J mice fed HFD for 20 weeks). AMO34a treatment was carried out by tail-vein injection three times over a 12-day period. Hepatic miR-34a levels, which were aberrantly elevated in the chronic HFD-fed mice before dosing, were rescued to levels seen in gender- and agematched lean mice after the full course of AMO34a treatment. The effects of AMO34a on miR-34a levels in other tissues were not reported. Notably, AMO34a treatment led to significant improvements in glucose tolerance and insulin sensitivity, matching levels observed in lean mice. Furthermore, silencing of miR-34a also significantly reduced serum levels of IL-6, insulin, and triglycerides, although levels of alanine transaminase (ALT) and aspartate transaminase (AST) were unaffected. These findings indicate that AMO34a treatment can correct the metabolic imbalance induced by chronic HFD without side effects of liver inflammation or other toxicities. A subsequent study in 2014 from the same group showed that suppression of miR-34a in adipose tissue reduces adiposity and improves metabolic phenotypes in diet-induced obese mice by promoting beige and brown fat formation [28]; however, these experiments were conducted with lentiviral inhibition constructs since AMOs are not as efficiently taken up by adipose compared to the liver.

Also in 2013, Kornfeld et al. [19] showed that miR-802 is significantly increased in the liver of two different mouse models of obesity and insulin resistance: (1) 8-12-week-old mice homozygous for a diabetes mutation in the leptin receptor gene $(d b / d b)$ and (2) 8-12-week-old mice fed HFD. They also demonstrated that hepatic miR-802 levels are significantly correlated with body mass index (BMI) in human subjects. These findings suggest that miR-802 may be linked to the development of systemic insulin resistance. Kornfeld et al. intravenously delivered small LNAs against miR-802 to HFD-fed mice on two consecutive days. Two weeks after the final dose, the authors observed remarkable suppression of miR-802 in the liver and kidney, but not in any other tissue. The inhibition of miR-802 led to a significant improvement in glucose tolerance, and this effect was not mediated by increased glucose uptake by the skeletal muscle or white adipose tissue, but rather enhanced insulin sensitivity in the liver (as measured by hepatic glucose production). miR-802 is relatively new on the miRNA scene, and much more remains to be uncovered about its functions in the liver as well as in other tissues where it is robustly expressed. For example, the effects of LNA-mediated suppression of miR-802 in the kidney have not yet been investigated. However, there is reason for cautious optimism regarding miR-802 as a compelling therapeutic target for obesity and insulin resistance.

\section{Hyperlipidemia and Type 2 Diabetes}

Hyperlipidemia refers to the chronic elevation of lipids (cholesterol and fat) in the bloodstream, which is a significant risk factor for a variety of metabolic diseases, including T2D. The condition is most commonly managed by modification of diet, as well as drugs such as statins and fibrates. However, these medications are not uniformly effective across individuals in the human population, and in some cases, long-term and/or high-dose usage could even lead to very harmful side effects such as severe muscle damage [29], which may not be curable if not detected and treated sufficiently early. Therefore, there remains a critical need for new, safe, and effective medications to combat hyperlipidemia. Anti-gene inhibitors are already in various stages of clinical testing for hyperlipidemia. For example, treatment with an anti-sense oligonucleotide inhibitor of the gene apolipoprotein C-III (APOC3), a critical regulator of plasma triglyceride levels, led to safe, dose-dependent, and long-term reductions in plasma triglycerides in phase II clinical trials [30].

In a recent study, Kurtz et al. [25] administered LNAs by tail-vein injection in 8-week-old wild-type C57BL6/J mice to inhibit miR-29 family members (LNA29) in vivo. One week post-dosing, the LNAs were most effectively taken up by the liver and kidney, and as in previous studies, the LNAs did not successfully cross the blood-brain barrier. Treatment with the LNA29 significantly reduced plasma triglyceride levels by $\sim 15 \%$ and cholesterol levels by $\sim 40 \%$, which is commensurate with the effects of statins, the most widely prescribed class of medications for hypercholesterolemia. The authors further demonstrated that the striking phenotypic effects of LNA29 were due in large part to suppression of lipogenic pathways in the liver. These findings raise the possibility that LNA29 could be used to lower cholesterol levels in diet-based or genetic animal models of hypercholesterolemia. Importantly, miR-29 has also been implicated in the control of insulin signaling [31], and therefore, inhibition of miR-29 may improve hepatic or even systemic insulin sensitivity as well, though this has not yet been demonstrated. Indeed, hepatic miR-29 is significantly elevated in a rat genetic model of diabetes and restored to normal levels upon treatment with Pioglitazone [32]. However, it should also be noted that miR-29 has also been shown to regulate a variety of other biological processes, including fibrosis [33, 34], immune response [35], and cell proliferation [36]. Therefore, future studies will need to assess comprehensively the systemic influences of AMO-mediated suppression of miR-29 and whether any potential side effects can be mitigated or circumvented with alternate dosing schemes or cocktail therapies.

In another study, $\mathrm{Ng}$ et al. [24] injected LNAs against miR24 (LNA24) weekly for 4 weeks into 16-week-old wild-type C57BL6/J mice that were fed a HFD for 8 weeks. After completion of the dosing scheme, miR-24 levels were dramatically 
reduced in the liver, although the efficacy of inhibition in other tissues was not reported. While the LNA24 treatment had no effect on the overall body or liver weight, it significantly reduced fat accumulation in both the liver and the plasma of the HFD-fed mice. The authors further demonstrated that the effect of LNA24 was mediated by increased expression and activity of the insulin induced gene 1 (Insig1) protein, which is responsible for sequestering in the endoplasmic reticulum the key transcription factor responsible for lipid production in the liver, sterol regulatory element binding protein 1 (Srebp1) [37]. Therefore, silencing of miR-24, as in the case of silencing of miR-29, leads to the suppression of hepatic lipogenesis, suggesting that LNA24 may be a therapeutic strategy for metabolic conditions in which lipid levels are pathologic. Recently, miR-24 was also identified as a critical regulator of pancreatic beta cell function [38], in part through direct regulation of Hnfla and Neurodl, which underlie the etiology of maturity-onset diabetes of the young (MODY). Furthermore, it was shown that suppression of aberrantly elevated miR-24 in pancreatic islets from HFD-fed mice significantly improves glucose-stimulated insulin secretion. These findings suggest that miR-24-based therapies may be relevant for T2D as well. Further studies are required to evaluate the effects of long-term dosing with LNA24, as well as to assess the efficacy of treatment in more severe metabolic disease models (e.g., long-term HFD).

\section{Other Conditions of Metabolic Dysfunction}

Some cardio-metabolic diseases quite different from T2D still share some features of metabolic dysfunction. One such disorder of growing public health concern is atherosclerosis, which is characterized by the buildup of arterial plaque (cholesterol, triglycerides, calcium, and other cellular debris) that causes the hardening and constriction of arteries, leading to vascular disease, coronary heart disease, stroke, and death. It is the most prevalent disease in the developed world and is predicted to be the number one cause of death worldwide by the year 2020 . Some medications, including those that lower plasma total cholesterol levels and improve lipoprotein profiles, can be used for treatment. However, the development of new, complementary therapeutic approaches for the regression of atherosclerosis remains an open and important area of biomedical investigation. The first and best-studied miRNA in the context of atherosclerosis is miR-33, which has two isoforms, miR-33a and miR$33 \mathrm{~b}$, encoded within the genes sterol regulatory element binding factor 2 (Srebf2) and sterol regulatory element binding factor 1 (Srebf1), respectively. The proteins encoded by these two genes are master transcriptional regulators of cholesterol and fatty acid synthesis [37]. In 2010, several independent studies used AMOs against miR-33 (AMO33) in mice to establish an important functional role for miR-33 in the maintenance of systemic cholesterol homeostasis [39-41]. Follow-up studies in mouse models of atherosclerosis (e.g., $\mathrm{Ldlr}^{-1-}$ ) produced conflicting reports about the safety and efficacy of long-term treatment $[42,43]$. However, in vivo studies in non-human primates appear to suggest that therapeutic silencing of miR33 is safe and atheroprotective, both in the short and long term $[44,45]$. The latter findings indicate that AMO33 could remain a candidate therapeutic strategy for atherosclerosis.

\section{Key Challenges in Therapeutic MicroRNA Silencing}

Despite the exciting and rapid pace of advance in the development and pre-clinical use of potent miRNA silencing technologies, there remain several major challenges for clinical development, three of which I will highlight here.

(1) The effects of long-term ( $>5$ weeks) AMO-mediated silencing of miRNAs are simply not known in most cases and require more thorough investigation. One notable exception is miR-33. While short-term $(<5$ weeks) silencing of miR-33 in mice was shown to be highly safe and efficacious by multiple groups, the data for longterm silencing has been conflicting. Although studies demonstrated that long-term silencing of miR-33 is safe in non-human primates, the mouse results provide caution enough to researchers that the effects of prolonged AMO treatment must be evaluated rigorously and comprehensively before proceeding to clinical trials.

(2) The use of larger animal models for pre-clinical studies is critical but still limited. While rodent-based models are among the most widely used, larger animals such as pigs and monkeys are more physiologically relevant from a therapeutic standpoint. Also, the extent to which rodent miRNA networks are conserved in humans is still an open question, and it is important to consider preclinical studies in animals that are less evolutionarily distant from humans. Rodent models are incredibly valuable, in part because of the availability of rich resources, and they will remain an important part of the fabric of translational science. However, it will be important to develop more resources for studies of larger animal models in order to gain confidence in the relevance of a particular AMO for potential clinical trials.

(3) AMO-based therapeutic silencing of miRNAs is currently heavily biased toward the liver (Table 1), in large part because the liver (as well as the kidney) is most effective at taking up circulating AMOs. While liver miRNAs contribute considerably to the governance of metabolic phenotypes, miRNAs in other tissues likely also have critical roles in the development of metabolic disease, such as miR-200a, which regulates pancreatic beta cell survival in type 2 diabetes [46•]; miR-375, which modulates insulin secretion from beta cells [47] and maintains alpha/beta cell 
mass in pancreatic islets [48]; and miR-7a, which controls the physiologic adaptation of beta cells to the metabolic conditions of obesity and type 2 diabetes [49]. Pancreatic islets and most other metabolic tissues such as adipose and skeletal muscle do not efficiently take up the type of AMOs currently in use. Alternate strategies are clearly needed in order to perform therapeutic silencing of miRNAs in tissues other than the liver and kidney.

\section{Conclusions and Future Outlook of Therapeutic MicroRNA Silencing}

A few different approaches have been suggested in the literature to expand therapeutic silencing of miRNAs beyond the liver. One strategy in particular holds substantial promise: aptamerAMO chimeras [50, 51••]. Specifically, an AMO can be chemically linked to an RNA aptamer, which is a structured RNA molecule designed to have high-affinity binding to a cellsurface receptor that is specific to a particular cell type. Once the aptamer-AMO chimera binds to the target receptor and is internalized, the link between the aptamer and the AMO is severed by Dicer, releasing the AMO in the cytoplasm. In this approach, the RNA aptamer essentially serves as a "guide" for the AMO to specific cell types. RNA aptamers can be selected for specific receptors of interest by high-throughput screening of large RNA libraries using strategies such as Systematic Evolution of Ligands by Exponential Enrichment (SELEX) [52]. Aptamer-siRNA chimeras have already been used effectively in xenografted mice to target particular oncogenes for knockdown only in cancer cells (using an aptamer designed for EpCAM, a cell surface antigen that is highly expressed in most epithelial cancers) in order to suppress tumor growth [53]. CD4 aptamer-siRNA chimeras have also been used to inhibit the transmission of human immunodeficiency virus (HIV) in humanized mice [54]. Aptamer-AMO chimeras represent the next frontier for cell-type specific therapeutic silencing of miRNAs in metabolic disorders, as well as a wider array of diseases.

\footnotetext{
Acknowledgments I would like to thank C. Lisa Kurtz for her help in surveying the literature for in vivo studies using AMOs and both C. Lisa Kurtz and Alisha Coffey for their review of the manuscript and helpful suggestions.

This work is supported in part by NIDDK/NIH (R01DK105965 awarded to PS) and in part by the American Diabetes Association (116-ACE-47 Pathway awarded to P.S.).

Compliance with Ethics Guidelines

Conflict of Interest Praveen Sethupathy declares that he has no conflict of interest.
}

Human and Animal Rights and Informed Consent This article does not contain any studies with human or animal subjects performed by any of the authors.
Open Access This article is distributed under the terms of the Creative Commons Attribution 4.0 International License (http:// creativecommons.org/licenses/by/4.0/), which permits unrestricted use, distribution, and reproduction in any medium, provided you give appropriate credit to the original author(s) and the source, provide a link to the Creative Commons license, and indicate if changes were made.

\section{References}

Papers of particular interest, published recently, have been highlighted as:

- Of importance

- Of major importance

1. Guay C, Regazzi R. Circulating microRNAs as novel biomarkers for diabetes mellitus. Nat Rev Endocrinol. 2013;9:513-21. doi:10. 1038/nrendo.2013.86.

2. Ivey KN, Srivastava D. MicroRNAs as regulators of differentiation and cell fate decisions. Cell Stem Cell. 2010;7:36-41. doi:10.1016/ j.stem.2010.06.012.

3. Dumortier O, Hinault C, Van Obberghen E. MicroRNAs and metabolism crosstalk in energy homeostasis. Cell Metab. 2013;18: 312-24. doi:10.1016/j.cmet.2013.06.004.

4. Ross SA, Davis CD. The emerging role of microRNAs and nutrition in modulating health and disease. Annu Rev Nutr. 2014;34: 305-36. doi:10.1146/annurev-nutr-071813-105729.

5. Archambaud $\mathrm{C}$ et al. The intestinal microbiota interferes with the microRNA response upon oral Listeria infection. mBio. 2013;4: e00707-00713. doi:10.1128/mBio.00707-13.

6. Vrijens K, Bollati V, Nawrot TS. MicroRNAs as potential signatures of environmental exposure or effect: a systematic review. Environ Health Perspect. 2015;123:399-411. doi:10.1289/ehp.1408459.

7. Boon RA, Vickers KC. Intercellular transport of microRNAs. Arterioscler Thromb Vasc Biol. 2013;33:186-92. doi:10.1161/ ATVBAHA.112.300139. This review provides a description of how circulating miRNAs may serve as intercellular messengers mediating organ-to-organ communication in health and disease.

8. van Rooij E, Kauppinen S. Development of microRNA therapeutics is coming of age. EMBO Mol Med. 2014;6:851-64. doi:10. 15252/emmm.201100899.

9. Fernandez-Valverde SL, Taft RJ, Mattick JS. MicroRNAs in betacell biology, insulin resistance, diabetes and its complications. Diabetes. 2011;60:1825-31. doi:10.2337/db11-0171.

10. Stenvang J, Petri A, Lindow M, Obad S, Kauppinen S. Inhibition of microRNA function by antimiR oligonucleotides. Silence. 2012;3: 1. doi:10.1186/1758-907X-3-1.

11. van Rooij E. The art of microRNA research. Circ Res. 2011;108: 219-34. doi:10.1161/CIRCRESAHA.110.227496.

12. van Rooij E, Purcell AL, Levin AA. Developing microRNA therapeutics. Circ Res. 2012;110:496-507. doi:10.1161/ CIRCRESAHA.111.247916.

13. Lanford RE et al. Therapeutic silencing of microRNA-122 in primates with chronic hepatitis $C$ virus infection. Science. 2010;327: 198-201. doi:10.1126/science.1178178.

14. Janssen HL et al. Treatment of HCV infection by targeting microRNA. N Engl J Med. 2013;368:1685-94. doi:10.1056/ NEJMoa1209026.

15. Frost RJ, Olson EN. Control of glucose homeostasis and insulin sensitivity by the Let-7 family of microRNAs. Proc Natl Acad Sci U S A. 2011;108:21075-80. doi:10.1073/pnas.1118922109.

16. Fu T et al. Aberrantly elevated microRNA-34a in obesity attenuates hepatic responses to FGF19 by targeting a membrane coreceptor 
beta-Klotho. Proc Natl Acad Sci U S A. 2012;109:16137-42. doi: 10.1073/pnas.1205951109.

17. Choi SE et al. Elevated microRNA-34a in obesity reduces NAD+ levels and SIRT1 activity by directly targeting NAMPT. Aging Cell. 2013;12:1062-72. doi:10.1111/acel.12135.

18.• Trajkovski M et al. MicroRNAs 103 and 107 regulate insulin sensitivity. Nature. 2011;474:649-53. doi:10.1038/nature10112. This is a seminal study on the role of $\mathrm{miR}-103 / 107$ in regulating insulin sensitivity in the liver and adipose in murine models of diabetes. These findings have led to the selection of miR-103/107 as a candidate for clinical trials (http://ir.regulusrx.com/ releasedetail.cfm?ReleaseID=905305).

19. Kornfeld JW et al. Obesity-induced overexpression of miR-802 impairs glucose metabolism through silencing of Hnflb. Nature. 2013;494:111-5. doi:10.1038/nature11793.

20. Grueter CE et al. A cardiac microRNA governs systemic energy homeostasis by regulation of MED13. Cell. 2012;149:671-83. doi: 10.1016/j.cell.2012.03.029.

21. Zhou B et al. Downregulation of miR-181a upregulates sirtuin-1 (SIRT1) and improves hepatic insulin sensitivity. Diabetologia. 2012;55:2032-43. doi:10.1007/s00125-012-2539-8.

22. de Guia RM et al. MicroRNA-379 couples glucocorticoid hormones to dysfunctional lipid homeostasis. EMBO J. 2015;34: 344-60. doi:10.15252/embj.201490464.

23. Ahn J, Lee H, Jung CH, Jeon TI, Ha TY. MicroRNA-146b promotes adipogenesis by suppressing the SIRT1-FOXO1 cascade. EMBO Mol Med. 2013;5:1602-12. doi:10.1002/emmm.201302647.

24. $\mathrm{Ng} \mathrm{R}$ et al. Inhibition of microRNA-24 expression in liver prevents hepatic lipid accumulation and hyperlipidemia. Hepatology. 2014;60:554-64. doi:10.1002/hep.27153.

25. Kurtz CL et al. Inhibition of miR-29 has a significant lipid-lowering benefit through suppression of lipogenic programs in liver. Sci Rep. 2015;5:12911. doi:10.1038/srep12911.

26. Foley NH, O'Neill LA. miR-107: a toll-like receptor-regulated miRNA dysregulated in obesity and type II diabetes. J Leukoc Biol. 2012;92:521-7. doi:10.1189/jlb.0312160.

27. Daimiel-Ruiz L et al. Dietary lipids modulate the expression of miR-107, a miRNA that regulates the circadian system. Mol Nutr Food Res. 2015;59:1865-78. doi:10.1002/mnfr.201570094.

28. Fu T et al. MicroRNA 34a inhibits beige and brown fat formation in obesity in part by suppressing adipocyte fibroblast growth factor 21 signaling and SIRT1 function. Mol Cell Biol. 2014;34:4130-42. doi:10.1128/MCB.00596-14.

29. Fernandez G, Spatz ES, Jablecki C, Phillips PS. Statin myopathy: a common dilemma not reflected in clinical trials. Cleve Clin J Med. 2011;78:393-403. doi:10.3949/ccjm.78a.10073.

30. Gaudet D et al. Antisense inhibition of apolipoprotein C-III in patients with hypertriglyceridemia. N Engl J Med. 2015;373:438-47. doi:10.1056/NEJMoa1400283.

31. Dooley J et al. The microRNA-29 family dictates the balance between homeostatic and pathological glucose handling in diabetes and obesity. Diabetes. 2016;65:53-61. doi:10.2337/db15-0770.

32. Kurtz CL et al. MicroRNA-29 fine-tunes the expression of key FOXA2-activated lipid metabolism genes and is dysregulated in animal models of insulin resistance and diabetes. Diabetes. 2014;63:3141-8. doi:10.2337/db13-1015.

33. Roderburg $\mathrm{C}$ et al. Micro-RNA profiling reveals a role for miR-29 in human and murine liver fibrosis. Hepatology. 2011;53:209-18. doi:10.1002/hep.23922.

34. He Y, Huang C, Lin X, Li J. MicroRNA-29 family, a crucial therapeutic target for fibrosis diseases. Biochimie. 2013;95:1355-9. doi:10.1016/j.biochi.2013.03.010.

35. $\mathrm{Ma} \mathrm{F}$ et al. The microRNA miR-29 controls innate and adaptive immune responses to intracellular bacterial infection by targeting interferon-gamma. Nat Immunol. 2011;12:861-9. doi:10.1038/ni. 2073.
36. Jiang H, Zhang G, Wu JH, Jiang CP. Diverse roles of miR-29 in cancer (review). Oncol Rep. 2014;31:1509-16. doi:10.3892/or.2014.3036.

37. Ye J, DeBose-Boyd RA. Regulation of cholesterol and fatty acid synthesis. Cold Spring Harb Perspect Biol. 2011;3. doi:10.1101/ cshperspect.a004754.

38. Zhu Y et al. MicroRNA-24/MODY gene regulatory pathway mediates pancreatic beta-cell dysfunction. Diabetes. 2013;62:3194 206. doi: $10.2337 / \mathrm{db} 13-0151$.

39. Najafi-Shoushtari SH et al. MicroRNA-33 and the SREBP host genes cooperate to control cholesterol homeostasis. Science. 2010;328:1566-9. doi:10.1126/science.1189123.

40. Rayner KJ et al. MiR-33 contributes to the regulation of cholesterol homeostasis. Science. 2010;328:1570-3. doi:10.1126/science. 1189862.

41. Marquart TJ, Allen RM, Ory DS, Baldan A. miR-33 links SREBP2 induction to repression of sterol transporters. Proc Natl Acad Sci U S A. 2010;107:12228-32. doi:10.1073/pnas.1005191107.

42. Goedeke $\mathrm{L}$ et al. Long-term therapeutic silencing of miR-33 increases circulating triglyceride levels and hepatic lipid accumulation in mice. EMBO Mol Med. 2014;6:1133-41. doi:10.15252/ emmm.201404046.

43. Marquart TJ, Wu J, Lusis AJ, Baldan A. Anti-miR-33 therapy does not alter the progression of atherosclerosis in low-density lipoprotein receptor-deficient mice. Arterioscler Thromb Vasc Biol. 2013;33:455-8. doi:10.1161/ATVBAHA.112.300639.

44. Rayner $\mathrm{KJ}$ et al. Inhibition of miR-33a/b in non-human primates raises plasma HDL and lowers VLDL triglycerides. Nature. 2011;478:404-7. doi:10.1038/nature10486.

45. Rottiers $\mathrm{V}$ et al. Pharmacological inhibition of a microRNA family in nonhuman primates by a seed-targeting 8 -mer antimiR. Sci Transl Med. 2013;5:212ra162. doi:10.1126/scitranslmed.3006840.

46. Belgardt BF, et al. The microRNA-200 family regulates pancreatic beta cell survival in type 2 diabetes. Nat Med. 2015;21: 619-27. doi: $10.1038 / \mathrm{nm} .3862$. This study describes the importance of a specific miRNA family in pancreatic beta cell survival and in the pathophysiology of diabetes. Options for therapeutic silencing of miRNAs in beta cells in vivo remain limited, and represent an important next frontier in research.

47. Poy $\mathrm{MN}$ et al. A pancreatic islet-specific microRNA regulates insulin secretion. Nature. 2004;432:226-30. doi:10.1038/nature03076.

48. Poy $\mathrm{MN}$ et al. miR-375 maintains normal pancreatic alpha- and beta-cell mass. Proc Natl Acad Sci U S A. 2009;106:5813-8. doi: 10.1073/pnas.0810550106.

49. Latreille $\mathrm{M}$ et al. MicroRNA-7a regulates pancreatic beta cell function. J Clin Invest. 2014;124:2722-35. doi:10.1172/JCI73066.

50. Wittrup A, Lieberman J. Knocking down disease: a progress report on siRNA therapeutics. Nat Rev Genet. 2015;16:543-52. doi:10. 1038/nrg3978.

51.• Lieberman J, Sharp PA. Harnessing RNA interference for therapy: the silent treatment. JAMA. 2015;313:1207-8. doi:10.1001/jama. 2015.1241. This review highlights the state-of-the-art in antisense oligonucleotide (ASO) technology for therapeutics. It includes a discussion of the RNA aptamer based strategy for targeted delivery of ASOs to specific tissues.

52. Tuerk C, Gold L. Systematic evolution of ligands by exponential enrichment: RNA ligands to bacteriophage T4 DNA polymerase. Science. 1990;249:505-10.

53. Gilboa-Geffen A et al. Gene knockdown by EpCAM aptamersiRNA chimeras suppresses epithelial breast cancers and their tumor-initiating cells. Mol Cancer Ther. 2015;14:2279-91. doi: 10.1158/1535-7163.MCT-15-0201-T.

54. Wheeler LA et al. Durable knockdown and protection from HIV transmission in humanized mice treated with gel-formulated CD4 aptamer-siRNA chimeras. Mol Ther. 2013;21:1378-89. doi:10. 1038/mt.2013.77. 